\title{
Suspected myocardial infarction and left bundle branch block: electrocardiographic indicators of acute ischaemia
}

\author{
J A Edhouse, M Sakr, J Angus, F P Morris
}

\begin{abstract}
Objectives-To examine the use of thrombolytic treatment in patients with suspected acute myocardial infarction (AMI) and left bundle branch block (LBBB). To evaluate electrocardiographic criteria for the identification of AMI in the presence of LBBB, and examine the implications of using these criteria in the clinical setting. Methods-A retrospective study over two years, based in two Sheffield teaching hospitals. Patients presenting with LBBB and suspected AMI were studied by analysis of an AMI database. The proportion of patients with $L B B B$ and AMI receiving thrombolysis, and the in-hospital delay before the start of treatment, were used as indicators of current performance.
\end{abstract}

Three predictive criteria were applied to the electrocardiograms (ECGs) retrospectively, and their ability to identify acute ischaemic change assessed. The implications of using the predictive criteria in the clinical setting were explored. Result-Twenty three per cent (5/22) of patients with LBBB and AMI did not receive thrombolysis, in the absence of documented contraindications. The mean in-hospital treatment delay for thrombolysed patients was 154 minutes. Forty eight per cent (16/33) of those thrombolysed did not have a final clinical diagnosis of AMI. In the majority of cases (8/12), the decision not to administer thrombolysis was based on a single ECG recording.

The presence of any of the predictive electrocardiographic criteria was associated with a diagnosis of AMI, with a sensitivity of 0.79 (95\% confidence interval 0.63 to 0.95 ), specificity 1 , positive predictive value 1 , and negative predictive value 0.79 . The $k$ scores between four independent observers showed either substantial or near perfect agreement.

Conclusion-Currently, thrombolytic treatment is under-utilised in patients with LBBB and AMI, and those who are thrombolysed endure lengthy delays before treatment. Patients with any of the predictive criteria should be throm- bolysed immediately. When the diagnosis is in doubt, serial ECGs may demonstrate evolving ischaemic change.

(F Accid Emerg Med 1999;16:331-335)

Keywords: bundle branch block; thrombolytic treatment; acute myocardial infarction

The electrocardiographic features of acute myocardial infarction are notoriously difficult to identify in the presence of left bundle branch block (LBBB), as the ST segment/T wave abnormalities in LBBB mimic the changes of acute ischaemia. Over the years many electrocardiographic criteria have been proposed to aid the identification of acute ischaemia in LBBB,${ }^{1-3}$ but most have proved insensitive and none have gained widespread acceptance.

The prognosis for acute myocardial infarction (AMI) with LBBB is worse than for AMI with normal ventricular conduction. ${ }^{4-7}$ Thrombolysis has been shown to significantly reduce the mortality rate in patients with $\mathrm{LBBB},{ }^{8-11}$ and the greatest benefits are seen in those treated early. ${ }^{812}$ It is recommended that patients with LBBB and symptoms consistent with AMI are managed in the same way as patients with ST segment elevation, on the basis that the LBBB is "presumably new", ${ }^{13}$ and this policy is well supported in the literature. ${ }^{1415}$ Nevertheless, there is evidence that thrombolysis is under-utilised in patients with LBBB. ${ }^{16}$

While the benefits of early thrombolysis are considerable, ${ }^{812}$ its potential complications are also well recognised. The most feared complication of thrombolysis is stroke, and the risk is highest in elderly patients, and those treated with tissue plasminogen activator. ${ }^{17}{ }^{18}$ It has been shown that about $50 \%$ of patients who present with chest pain and LBBB are not infarcting, ${ }^{1}$ and this figure may be as high as $70 \% .{ }^{19}$ It would be ideal to avoid exposing these non-infarcting patients to the risks of thrombolytic drugs.

Sgarbossa et al proposed three electrocardiographic criteria to identify acute ischaemia in the presence of LBBB (see table 1); each criterion has a weighted score, and an algorithm indicates the overall probability of AMI ranging between $16 \%$ and $100 \% .^{20}$

These criteria illustrate a characteristic feature of the electrocardiogram (ECG) in LBBB: abnormal ventricular depolarisation is associated with secondary alteration of the recovery process, with the result that the ST segment and $T$ waves are discordant with the 


\begin{tabular}{lll}
\hline & $\begin{array}{ll}L B B B \\
(n=50)\end{array}$ & $\begin{array}{l}\text { ECGs without } \\
\text { LBBB } \\
(n=747)\end{array}$ \\
\hline Mean age (years) & 71 & 66 \\
No eligible for thrombolysis & 45 & 720 \\
No of eligible patients receiving thrombolysis (\%) & $33(73)$ & $688(95.5)$ \\
Door to needle time (minutes) & & 79 \\
Mean & 154 & 60 \\
Median & 121 & $10-643$ \\
Range & $32-449$ & \\
\hline
\end{tabular}

main QRS deflection. ST segment elevation in the same direction as that of the QRS complex is not expected in uncomplicated LBBB, and its presence signifies acute ischaemia. ${ }^{1}$

The purpose of our study was to examine the current local management of patients presenting with chest pain and LBBB, to evaluate the predictive electrocardiographic criteria, ${ }^{20}$ and to assess their potential effect on patient care.

\section{Methods}

We performed a retrospective study over a two year period based in two Sheffield teaching hospitals. All patients presenting with ischaemic sounding chest pain were identified prospectively and followed up until discharge, and those eligible for thrombolytic treatment were entered on a database.

Patients presenting with suspected AMI and complete LBBB were studied retrospectively. Complete LBBB was defined as: (1) QRS duration of $>0.120$ seconds in the presence of a supraventricular rhythm; (2) broad and notched or slurred $R$ in I and V5 or V6; (3) absence of $Q$ wave in I and V5 and V6; (4) $R$ wave peak time $>0.060$ seconds in V5 or V6. ${ }^{21}$

We noted the following:

- Whether thrombolytic treatment was administered.

- If thrombolysis was not administered, any reason recorded in the case notes.

- The interval between arrival at hospital and start of thrombolysis, or "door to needle" time.

- The results of serum creatine kinase estimations, on days 1,2 , and 3 .

All ECGs recorded during admission were obtained. A diagnosis of AMI was established on the basis of a peak creatine kinase value of at least $400 \mathrm{IU} / 1$, or if unavailable, on the results of postmortem examination. Other serum markers of myocardial injury were not collected routinely.

The presenting ECGs were reviewed blindly, and the clinical prediction rule ${ }^{20}$ was used to estimate the probability of AMI, each patient being awarded a score between 0 and 10. The actual clinical diagnosis was then compared

Table 3 Administration of thrombolysis

\begin{tabular}{llll}
\hline & $A M I^{*}$ & No AMI & $\begin{array}{l}\text { No of } \\
\text { patients }\end{array}$ \\
\hline Thrombolysis given & 17 & 16 & 33 \\
Thrombolysis withheld & 5 & 7 & 12 \\
Thrombolysis contraindicated & 2 & 1 & 3 \\
Presented $>12$ hours after onset chest pain & 2 & 0 & 2 \\
Total & 26 & 24 & 50 \\
\hline
\end{tabular}

${ }^{\star} \mathrm{AMI}$ indicated by serum creatine kinase level $>400 \mathrm{IU} / \mathrm{l}$.
A

III
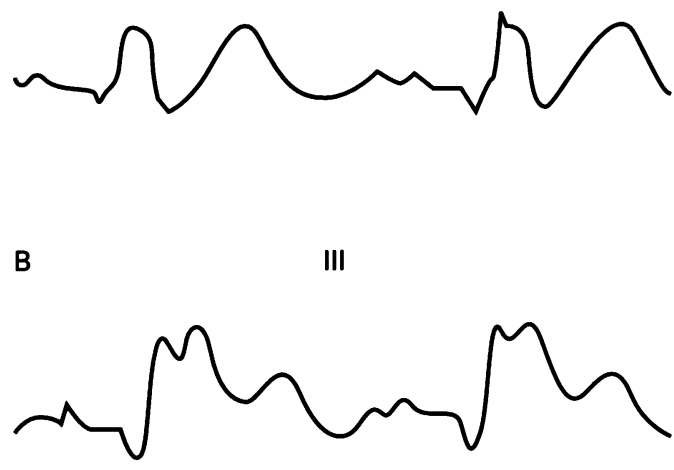

Figure 1 Serial ECG changes in patients with biochemical evidence of $A M I$. (A) Lead III recorded at 0345 hours; (B) lead III recorded at 0502 hours, with ST segment elevation of $>1 \mathrm{~mm}$.

with the clinical prediction rule score. Interobserver agreement was tested by calculation of $\kappa$ scores for four independent observers (A\&E consultants and specialist registrars).

\section{Results}

In total, 797 patients were admitted with suspected AMI and had electrocardiographic criteria suitable for thrombolysis. LBBB was present on or shortly after admission in 50 presentations of 49 patients (see table 2). Cardiac enzyme estimations were available on 49/50 occasions; one patient died shortly after admission, and the diagnosis was based on postmortem examination findings. A final diagnosis of AMI was made in 52\% (26/50) of cases. Two patients presented more than 12 hours after the onset of chest pain and their ECGs were excluded from further analysis.

ADMINISTRATION OF THROMBOLYTIC TREATMENT TO ELIGIBLE PATIENTS

Forty five patients with LBBB were eligible for thrombolysis, and $73 \%$ (33/45) of these received it (see table 3). The mean delay between arrival at hospital and the start of thrombolysis was 154 minutes. Five patients with AMI and LBBB did not receive thrombolysis. Forty eight per cent $(16 / 33)$ of thrombolysed patients had no biochemical evidence of AMI.

SERIAL ECGS

Twelve patients with $\mathrm{LBBB}$ did not receive thrombolysis; eight of these had only one ECG recorded on the day of admission. Serial ECGs were recorded in nine patients with a final diagnosis of AMI; in five cases the ECGs demonstrated evolving ischaemic change (see figs 1-3).

COMPLICATIONS OF THROMBOLYTIC TREATMENT One patient with AMI suffered a haemorrhagic stroke after thrombolysis. Minor haemorrhagic complications occurred in two patients, one of whom had no biochemical evidence of AMI.

NEW ONSET LBBB

Three patients developed LBBB shortly after admission, and consequently all received thrombolysis. None of these patients had 
A

V1

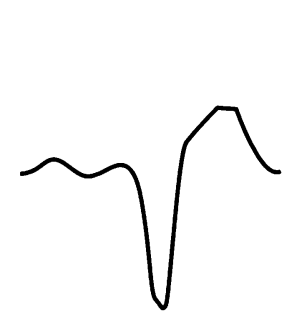

B
V2
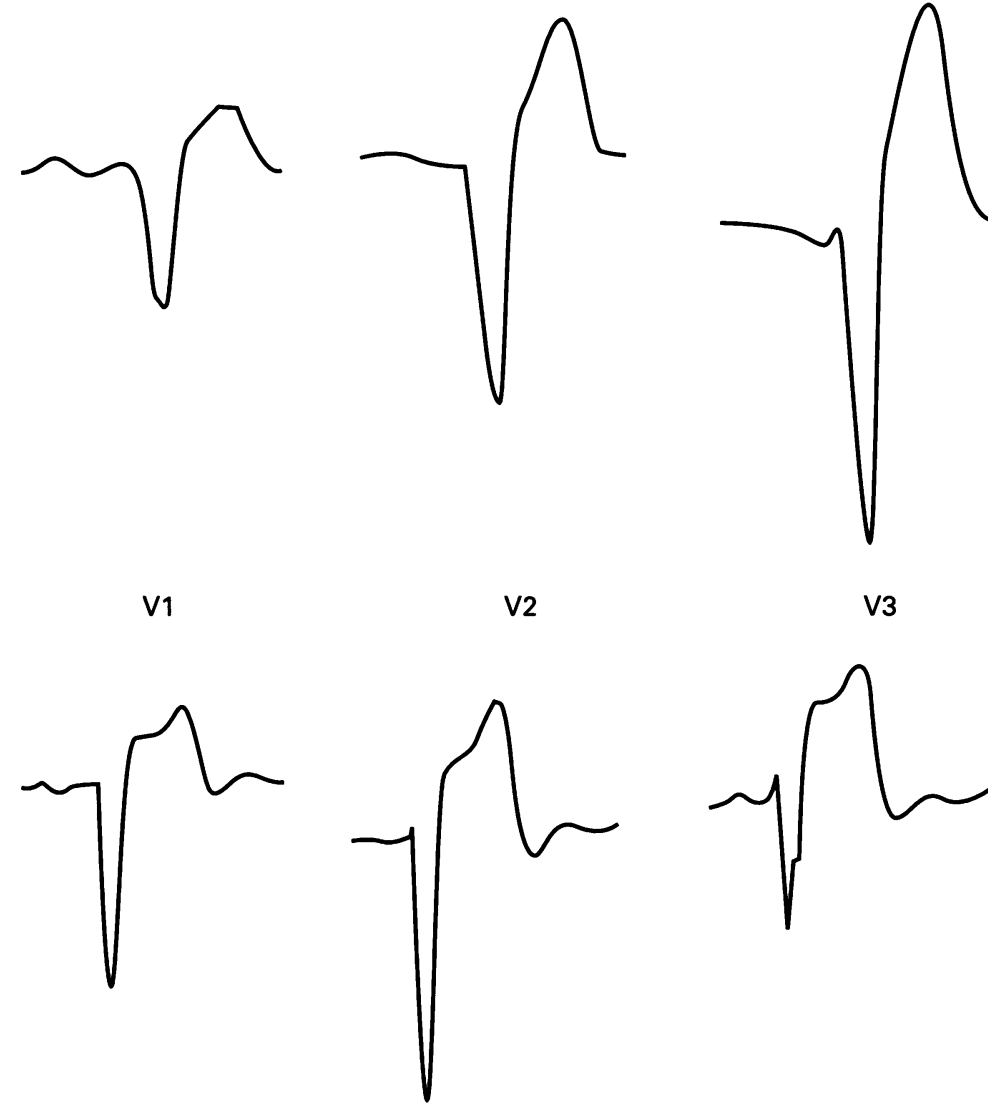

Figure 2 Serial ECG changes in patients with biochemical evidence of $A M I$. $(A)$ Leads $V 1, V 2, V 3$ recorded at 0538 hours; (B) leads V1,V2,V3 recorded at 0736 hours, showing evolving $S T$ segment elevation.

biochemical evidence of AMI, and one patient subsequently exhibited frequent bouts of intermittent LBBB, interspersed with periods of normal conduction.

APPLICATION OF THE CLINICAL PREDICTION RULE The ECGs of 48 patients were used to test the clinical prediction rule; the prevalence of AMI in the test group was 0.5. Blinded to the final clinical diagnosis, each patient was allocated a score indicating their probability of AMI using the published algorithm. ${ }^{20}$

Two clear groups emerged (see fig 4); all patients (24/24) with no biochemical evidence of AMI were found to have a clinical prediction rule score of 0 . Of those with a proved AMI, $79 \%(19 / 24)$ of the ECGs had a prediction rule score of 2 or more.

\section{SENSITIVITY, SPECIFICITY, AND PREDICTIVE} VALUES

In our study, the presence of any of the three predictive electrocardiographic criteria was associated with a diagnosis of AMI. With none of the predictive criteria on the initial ECG there was still a $27 \%$ probability of an AMI. Serial ECGs were performed before thrombolysis in five of the patients who infarcted; when these were also examined, the probability of an AMI with a persisting score of 0 fell to $17.2 \%$, similar to the figure of $16 \%$ quoted by Sgarbossa et al. ${ }^{20}$
To accurately assess the performance parameters of the test we therefore examined serial ECGs when available, and used a score $>0$ to indicate AMI. A score $>0$ was found to be diagnostic of AMI with a sensitivity of 0.79 ( $95 \%$ confidence interval 0.63 to 0.95 ), and specificity 1 , positive predictive value $100 \%$, and negative predictive value $79 \%$.

INTEROBSERVER AGREEMENT

Four independent observers were asked to indicate whether any of the predictive ECG criteria were present. There was either substantial or near perfect agreement between the observers as indicated by $\kappa$ scores of 0.65 , $0.69,0.74,0.77,0.81$, and 0.87 .

\section{Discussion}

The detection of ischaemic change in the presence of LBBB is an old problem, but there is new pressure to find a solution. Thrombolytic treatment confers a significant mortality reduction in patients with LBBB and AMI, ${ }^{8-11}$ and is of particular benefit if given early. ${ }^{812}$

The administration of thrombolysis to all patients with LBBB and suspected AMI can be justified by the absolute net mortality reduction seen when this group is treated. ${ }^{81011}$ Nevertheless, our study and others ${ }^{1}$ suggest that half of these patients not infarcting, and it would be ideal to minimise the exposure of patients without AMI to the risks of thrombolytic drugs.

\section{CURRENT GUIDELINES}

It is recommended that patients with $\mathrm{LBBB}$ and suspected AMI are managed in the same way as patients with ST segment elevation, on the basis that the LBBB is "presumably new". ${ }^{13}$ Our evidence and that of other studies ${ }^{162}$ shows that in practice these guidelines are not adhered to, and thrombolysis remains underutilised in patients with LBBB, both in the United States and the UK. Doctors administering thrombolysis are extremely concerned about its potential side effects. ${ }^{23}$ The underuse of thrombolysis may reflect a reluctance by doctors to administer the drug without a confident diagnosis of AMI.

Patients with LBBB have much longer door to needle times compared with other thrombolysed patients. This may reflect attempts to target treatment at those with "presumably new" LBBB, by searching for old ECGs before thrombolysis. However, patients with longstanding LBBB may present with a new infarct. Even if an old ECG does not show LBBB, the abnormality may have developed at any time in the intervening period. Some patients with demonstrably new onset LBBB may not be acutely infarcting, but merely exhibiting intermittent LBBB, as in our study. Thus, basing the decision to thrombolyse on the appearance of old ECGs has many practical difficulties.

The diagnostic value of dynamic ECG changes in LBBB has been previously highlighted, ${ }^{23} 24$ but it appears that serial ECGs are still not performed routinely. We found that serial ECGs, even though 
A
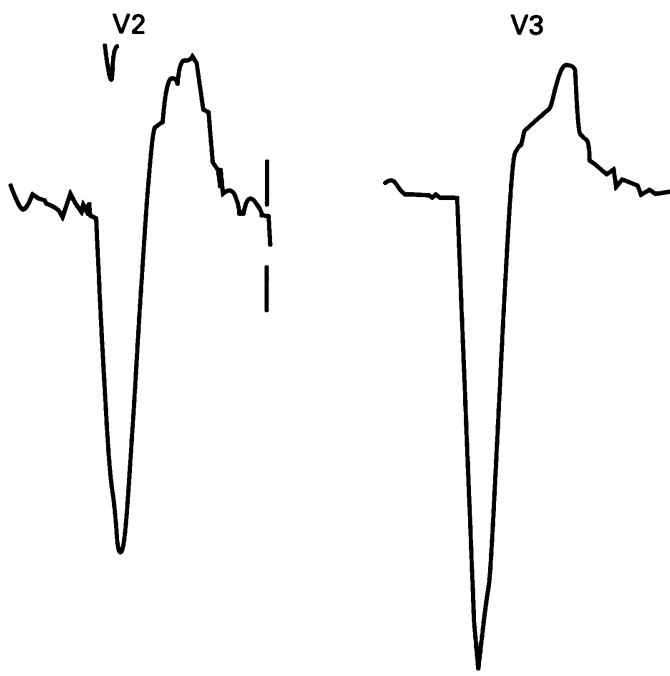

B V2

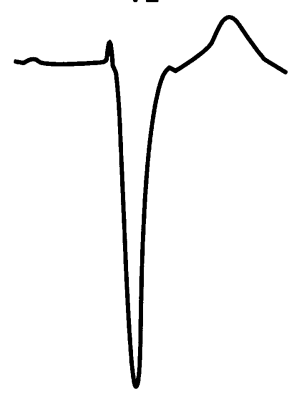

V3

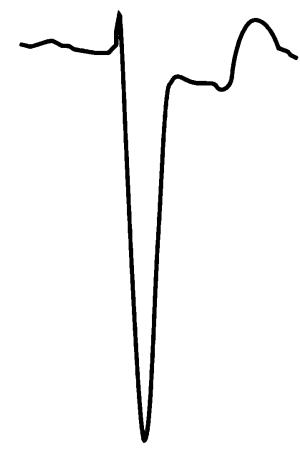

Figure 3 Serial ECG changes in patients with biochemical evidence of $A M I$. (A) Leads V2 and V3 recorded at 1454 hours; $(B)$ leads V2 and V3 recorded at 1508 hours, showing ST segment depression.

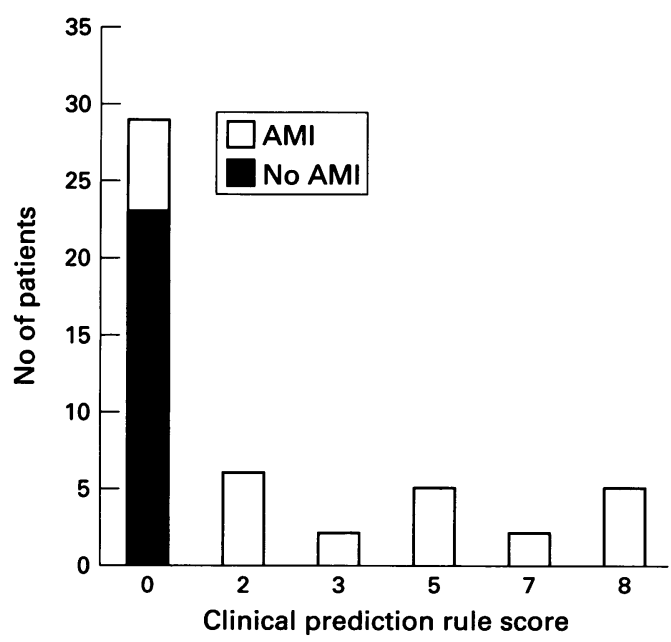

Figure 4 Scores based on flow chart for the prediction of $A M I$ in the presence of $L B B B$.

performed in a minority of cases, demonstrated evolving ischaemia in a high proportion of infarcting patients.

EFFECT OF CLINICAL PREDICTION RULE ON MANAGEMENT OF PATIENTS WITH CHEST PAIN AND LBBB

In this study, the presence of any of the three predictive electrocardiographic criteria was diagnostic of AMI. Patients presenting with

any of these criteria should be thrombolysed immediately; searches for old ECGs are unnecessary. A knowledge of these criteria could lead to significant reductions in door to needle time for some patients.

A patient with an initial ECG score of 0 has a $27 \%$ chance of having an AMI. Serial ECGs or continuous ST segment monitoring will identify a significant proportion of the remaining patients who are infarcting, ${ }^{324}$ and are essential whenever the diagnosis is in doubt. It is likely, however, that a minority of patients with AMI will not develop the predictive electrocardiographic criteria; the exact proportion is unknown, since neither the original work ${ }^{20}$ nor recent attempts at validation ${ }^{19}$ have examined the accuracy of the criteria using a series of ECGs.

\section{LIMITATIONS OF THIS STUDY}

This study has the usual limitations of a retrospective study. Since often only one ECG, rather than a series, was available for testing the criteria, their sensitivity may have been underestimated. Serial creatine kinase values are a relatively crude indicator of the presence of AMI; the number of AMIs in the group may have been underestimated. Though the reasons for withholding thrombolysis were often unclear retrospectively, it seemed reasonable to assume that if specific contraindications had been present they would have been documented in the case notes.

\section{Conclusion}

Thrombolytic treatment significantly reduces mortality in AMI with LBBB, particularly if given early, and the magnitude of the benefit justifies the administration of thrombolysis to all patients with LBBB and suspected AMI. However, thrombolysis remains under-utilised in patients with LBBB, and those who do receive it have long door to needle times. The under-utilisation of thrombolysis and long door to needle times may reflect a reluctance to expose patients to the risks of thrombolytic drugs without a confident diagnosis of AMI.

Attempts to validate the electrocardiographic criteria have so far been flawed, and a prospective study, using serial ECGs when appropriate, is needed to establish their true sensitivity. On the strength of current evidence, the decision to withhold thrombolysis should not be based solely on the absence of these criteria.

The described criteria can reliably identify the majority of patients with AMI. The presence of any of the criteria in a patient with LBBB and suspected AMI warrants immediate thrombolysis; searches for old ECGs unnecessarily prolong the door to needle time. Application of the criteria could significantly reduce in-hospital treatment delays for many patients. Patients in whom the diagnosis is in doubt require serial ECGs in case of evolving ischaemic change.

The authors would like to acknowledge the cooperation of $\mathrm{Dr} \mathrm{K}$ Channer and Dr S Campbell and all the consultant cardiologists and physicians of the Northern General and Royal Hallamshire Hospitals, and the coronary care unit staff of the Northern General and Royal Hallamshire Hospitals. 
We would also like to acknowledge the assistance of the audit departments of the Northern General and Royal Hallamshire Hospitals, and Melloney Ferrar for her help with data collection.

Conflict of interest: none.

Funding: $M$ Sakr and J Angus were supported by Sheffield Health Authority.

1 Hands ME, Cook EF, Stone PH, et al. Electrocardiographic diagnosis of myocardial infarction in the presence of complete left bundle branch block. Am Heart F 1988;116:23 30.

2 Wackers FJ. The diagnosis of myocardial infarction in the presence of left bundle branch block. Cardiol Clin 1987;5:393-401.

3 Fesmire FM. ECG diagnosis of acute myocardial infarction in the presence of left bundle-branch block in patients undergoing continuous ECG monitoring. Ann Emerg Med 1995;26:69-82.

4 Hindman MC, Wagner GS, Jaro $M$, et al. The clinical significance of bundle branch block complicating acute myocardial infarction. 1. Clinical characteristics, hospital mortality, and one-year follow-up. Circulation 1978:58: 679-88.

$5 \mathrm{Col} \mathrm{JJ}$, Weinberg SL. The incidence and mortality of intraventricular conduction defects in acute myocardial infarcventricular conduction defects in acute

6 Steinmetz E, Haghfelt T, Thygesen K. Incidence and prognosis of intraventricular block in acute myocardial infarcnosis of intraventricular block in
tion. Cardiology 1979;64:280-8.

7 Fazzini PF, Marchi F, Pucci P. Prognostic value of intraventricular conduction blocks in acute myocardial infarction tricular conduction blocks in

8 ISIS-2 Collaborative Group. Randomised trial of intravenous streptokinase, oral aspirin, both, or neither among 17187 cases of suspected acute myocardial infarction ISIS-2. Lancet 1988;ii:349-60.

9 Newby KH, Pisano E, Krucoff MW, et al. Incidence and clinical relevance of the occurrence of bundle-branch block in patients treated with thrombolytic therapy. Circulation 1996;94:2424-8.

10 Sgarbossa EB, Pinski SL, Topol EJ, et al. Acute myocardial infarction and complete bundle branch block at hospital admission: clinical characteristics and outcome in the thrombolytic era. $₹$ Am Coll Cardiol 1998;31:105-10.

11 FTTCG Fibrinolytic Therapy Trialists' Collaborative Group. Indications for fibrinolytic therapy in suspected acute myocardial infarction: collaborative overview of early mortality and major morbidity results from all randomised trials of more than 1000 patients. Lancet 1994;343:311-22.
12 Rawles $J$ on behalf of the GREAT group. Halving of mortality at 1 year by domiciliary thrombolysis in the Grampian region early anistreplase trial (GREAT). $\mathcal{F} \mathrm{Am}$ Coll Cardiol 1994;23:1-5.

13 Ryan TJ, Anderson JL, Antman EM, et al. ACC/AHA guidelines for the management of patients with acute myocardial infarction: executive summary. Circulation 1996;94: 2341-50.

14 Wellens HJJ. Acute myocardial infarction and left bundlebranch block-can we lift the veil? N Engl f Med 1996;334: 528-9.

15 Habib GB. Current status of thrombolysis in acute myocardial infarction. Optimal utilization of thrombolysis in clinical subsets. Chest 1995;107:528-34.

16 Barron HV, Bowlby LJ, Breen T, et al. Use of reperfusion therapy for acute myocardial infarction in the United States. Data from the national registry of myocardial infarction 2. Circulation 1998;97:1150-6.

17 The GUSTO investigators. An international randomized trial comparing four thrombolytic strategies for acute myocardial infarction. N Engl f Med 1993;329:673-82.

18 Maggioni AP, Franzosi MG, Santoro E, et al. The risk of stroke in patients with acute myocardial infarction after thrombolytic and antithrombotic treatment. $N$ Engl $f \mathrm{Med}$ 1992;327:1-6.

19 Shlipak MG, Lyons WL, Go AS, et al. Should the electrocardiogram be used to guide therapy for patients with left bundle-branch block and suspected myocardial infarction? fAMA 1999;281:714-19.

20 Sgarbossa EB, Pinski SL, Barbagelata A, et al. The GUSTO-1 Investigators. Electrocardiographic diagnosis of evolving acute myocardial infarction in the presence of left bundle-branch block. $N$ Engl $f$ Med 1996;334:481-7.

21 Willems JL, Robles de Medina EO, Bernard R, et al. World Health Organisation/International Society and Federation for Cardiology Task Force Ad Hoc. $\mathcal{f} \mathrm{Am}$ Coll Cardiol 1985;5:1261-75.

22 Ketley D, Woods KL for the European Secondary Prevention Study Group. Selection factors for the use of Prevention Study Group. Selection factors for the use of thrombolytic treatment in acute myocardial infarction: a Kingdom. Br Heart $\mathcal{F}$ 1995;74:224-8.

23 Wald DS. Perceived contraindications to thrombolytic treatment in acute myocardial infarction. A survey at a teaching hospital. F Accid Emerg Med 1998;15:329-31.

24 Brady WJ Jr, Aufderheide TP. Left bundle-branch block pattern complicating the electrocardiographic evaluation of acute myocardial infarction. Acad Emerg Med 1997;4:5662. 